head resection is still accepted as treatment of choice in most instances. Unstable distal ulnar stumps and loss of power grip raised the wish for an alternative. There is a new distal ulnar head prosthesis on the marked, which might be an possible implant for the future for selected cases.

On the level of the finger joints Swansons silastic implants, also in newer different designs, are still the implants of choice for most surgeons. High implant failure rate and the wish to go away from Silastic as implant material, motivated different authors for new implant designs and the use of newer materials. Despite these efforts a real breakthrough as well in design as in materials is still somewhat missing. Carbon fibre based materials or ceramics might have a good potential for the future.

Besides the implant design and the material, the understanding of soft tissue pathomechanics and its consequences for prosthetic replacement is raising. Ligament reconstruction and possible use of artificial ligaments might be a concept for the future.

The lecture should give an understanding of the pathomechanics of rheumatoid arthritis and its consequences for prosthetic replacement. It should give an overview of current prosthetic designs and their clinical results and provide an outlook of new concepts of prosthetic replacement and future development.

\section{SP0108 PRESENT STATUS OF TALOCRURAL ARTHROPLASTY IN RHEUMATOID ARTHRITIS}

F-W Hagena. Auguste-Viktoria-Klinik, Bad Oeynhausen, Germany

\subsection{6/annrheumdis-2001.48}

The new approach of interdisciplinary management of the multiple joint disease acknowledges the distinction between interpretation of the systemic and of the local activity. The systemic inflammatory disease may be modified or controlled by effective drug protocols. This does not prevent or exclude local inflammation or progressive alterations of various joint in the ,malignant forms of RA. In this respect rheumatoid arthritis surgery surves as local augmentation for the systemic therapy to prevent disability and/or to restore function.

An involvement of the talocrural and of the subtalar joint is reported in up to $50 \%$ in RA. At the same time the ipsilateral knee joint shows a local activity in $60 \%$ of the RA-patients.

Depending on the degree of the local activity and on the degree of deterioration the concept of rheumatoid surgery in the early phase includes the irradication of the inflamed tissue in order to reduce the progress of pain and joint damage. In the later phase related to the Larsen, Dale, Eek radiographic stages $>$ III reconstructive surgery of the talocrural joints is demanded to reduce the impairment and to restore the daily activities of the patients. To reduce pain, to stabilise and to realign the foot in neutral position ankle arthroplasties and arthrodesis are alternative procedures.

The function of the ankle joint extremity considerably influences the other joints of the lower. Stiffness and ankylosis of the ankle joint reduces the functional activity of the lower extremity. The stress distribution at the lower limb may alter the adjacent joints if the ankle joint is arthrodesed.

In former years the outcome of ankle arthroplasties has been unpredictable. The results following total replacement of the ankle using the ICLH-, TRP- or the Mayo ankle prostheses (published before 1996) reported an incidence of $22 \%$ to $75 \%$ aseptic loosening with a follow-up of 3 to 9 years. Only the experience with a follow-up of 5 years in 66 RA-patients with the modified TRP-arthroplasty reported by Pahle et al., 1987 showed good results in $83 \%$ of the patients. $1.5 \%$ of the patients had bad results after this observation time.

With new "near anatomic" designs of total ankle prostheses in a comparative study no significant difference had been observed for the OA-group $72.7 \%$ and for the RA-group $75.5 \%$ at 14 years (Kofoed, Sorensen 1998). A cumulative survival rate of the ankle replacement at 6 years of $94.3 \%$ has been reported by Schill et al., 1998. Evenmore it has been shown that in comparison to an ankle arthrodesis the total ankle replacements give significantly better pain relief, better function and a lower infection rate without development of subtalar arthrosis. Especially for patients with a multiple joint involvement in RA it is of great importance that after the total ankle arthroplasty a normal weight bearing is allowed already two weeks after implantation. In comparison to this fact ankle arthrodesis needs reduced weight bearing for at least 6 to 8 weeks.

Pain relief is achieved in more than $90 \%$ of the patients after an average follow-up time of more than 3 years. The implantation technique and the implants have been improved. Most of the available designs offer a cementless implantation of the ankle arthroplasties.

The functional results show an increased motion after total ankle replacement.

It is very important to exclude those patients with severe osteonecrosis of the talus or/and of the distal tibia to avoid early migration and the potential of early loosening.

Conclusion We like to emphasise that it seems appropriate today that in case of deterioration of the ankle joint in RA the indication for a total ankle replacement is preferable and the results are superior to an ankle arthrodesis.

\section{Biology and the destruction of cartilage/ bone - Saturday 16 June, 12.00-13.30/ Congress Hall}

\section{SP0109 HOW USEFUL ARE CONVENTIONAL DMARDS AND WHEN SHOULD BIOLOGICS, IN PARTICULAR TNF BLOCKING AGENTS, BE USED?}

L Van de Putte. Rheumatology, University Medical Centre Nijmegen, Nijmegen, The Netherlands

\subsection{6/annrheumdis-2001.49}

Rheumatoid arthritis is a chronic, systemic, inflammatory disease with polyarthritis as the most dominant clinical sign. Pharmacotherapy is, increasingly, the corner stone of treatment. Conventional drug treatment consists of NSAIDs, (combinations of) DMARDs and corticosteroids.

In recent years dramatic changes have occurred in the treatment of the disease. These can be categorised as follows:

- Early treatment: it has become clear that (irreversible) joint damage occurs relatively early in the disease course. In addition recent studies have indicated that more advanced stages of the disease are more difficult to treat.

- More and more effective DMARDs. Some of these DMARDs have a relatively fast mode of action, allowing for more adequate drug titration towards efficacy. Another interesting 
development is the attempt towards side effect reduction. Successful examples are comedication with folic acid during methotrexate treatment and of biphosphonate during corticosteroid treatment.

- Target oriented therapies: whereas anti-T cell therapies so far have not been consistently successful, TNF blocking agents have revolutionised treatment of rheumatoid arthritis. These agents work fast and give a good response in over half of the patients. In addition it has been shown that these therapies have the potential of stopping (radiographic) joint damage. From a practical point of view there are at least a few problems: these therapies are rather expensive and long term follow-up data are not yet available. Especially the latter is important, since TNF plays an important role in inflammation and immunity and thefore chronic suppression may also have negative consequences. Furthermore, it is advised that during TNF blocking therapies, doctor and patiënt should be alert on the presence or development of infectious diseases.

In view of the above considerations, current use of TNF blocking therapies should be restricted to patiënts with active disease, despite adequate DMARD therapy, including methotrexate. Possible future strategies will be discussed.

\section{SP0112 EXPERIENCES FROM A NATIONAL SURVEILLANCE PROGRAM FOR EFFECTS, SIDE-EFFECTS AND COSTS OF TNF-BLOCKING AGENTS IN SWEDEN}

N Feltelius. Division of Clinical Trials, Medical Products Agency, Uppsala, Sweden

10.1136/annrheumdis-2001.50

The introduction of TNF-blockers in rheumatology has focused on the need for new systems for follow-up of new drugs. Many drugs, like the TNF-blockers have new mechanisms of actions and thus unknown side effects. The national influence on the approval of new drugs has decreased with the common European regulatory system. However the economic consequences of these often expensive treatments are to be handled on the national level. Thus for medical as well as economical reasons national efforts to collect information about recently marketed drugs are important.

In a joint project the Swedish Medical Products Agency (MPA) and The Swedish Society for Rheumatology introduced a follow-up system for the new TNF-blockers when used in rheumatoid arthritis. The system was implemented already before marketing. The aim is to elaborate a system for collecting and reporting pharmacotherapy data from all rheumatology units in Sweden to assess safety and efficacy. This is to optimise treatment but also to form the basis for future surveillance programmes for other new drugs.

The study started in early 1999 with a planned 2-year duration. Assessments of patients are scheduled at 0,3, 6, 12, 18 and 24 months. Patients included are those with active RA who has failed previous DMARD treatment. All patients are treated on a named-patient-basis and are identified by a unique personal number. This system makes possible future cross-linking with other health data bases. Disease activity is measured according to EULAR core set. The concomitant use of DMARDs and other medications is recorded in detail.

Treatment reports have till now been collected from 1280 patients. The overall clinical outcome of etanercept as well as of infliximab treatment is much in line with what has been reported from randomised controlled studies (RCTs). Only a small number of patients has been followed for over 12 months but efficacy seems sustained also after this time point.

Reporting of adverse events (AEs) is higher in this surveillance study than what can be expected from the regular spontaneous reporting system. In total 425 AEs have been reported in the cohort. Most frequent are injection site reactions and other dermatological manifestations. Among serious adverse events infections were predominant, with cases of septic arthritis and septicaemia reported. The cost for TNF-blocker treatment in Sweden during the year 2000 is 170 million SK. The spending calculated per inhabitant indicate substancial regional differences in prescription. These figures includes treatment of Crohn's disease patients.

The main conclusions from this study are: 1 . Results of TNFblocker treatment from RCTs seem valid also in an everyday clinical setting. 2. Reporting of adverse events can be substantially improved compared to the existing systems. 3 . The logistics of reporting was the main problem in establishing the data collecting system. 4. The involvement of the MPA and the Swedish Society for Rheumatology was necessary to implement the system. 5. For the future the establishing of firm and independent systems that can further develop these kind of drug surveillance is highly desirable. In this struggle the participation of all key players regulatory agencies, professional societies, academic institutions and drug companies is necessary.

\section{Metabolic disorders and the musculoskeletal system - Saturday 16 June, 12.00-13.30/ Forum Hall}

\section{SP0115 CRYSTALS AND JOINTS}

M Doherty. Academic Rheumatology, City Hospital, Nottingham, UK

10.1136/annrheumdis-2001.51

Primary metabolic disease may predispose to the deposition of a variety of crystals in and around joints, most notably calcium pyrophosphate (CPPD) and apatite. Crystal associated musculoskeletal symptoms may be the presentation of such disease. Renal failure additionally predisposes to urate and oxalate crystal deposition. In most disease states the principal mechanisms relate to elevated ionic products.

There is good evidence that hyperparathyroidism, hypomagnesaemia, haemochromatosis and hypophosphatasia predispose to CPPD deposition; data are less convincing for hypothyroidism, Wilson's disease and other putative associations. Clinical features are acute "pseudogout" attacks in association with polyarticular chondrocalcinosis (CC). Haemochromatosis is exceptional in additionally causing structural joint change and chronic symptoms. Patients $<55$ years presenting with recurrent acute attacks and polyarticular CC should be screened for metabolic disease (minimum $=$ serum ferritin, magnesium, calcium, alkaline phosphatase). Treatment of the primary disease, however, probably little influences the outcome of CPPD and CC. All these diseases increase extracellular pyrophosphate levels; effects on CPPD crystal nucleation and dissolution, however, are also evident. Rare monogenic familial CC may shortly be explained as primary metabolic disorders of pyrophosphate metabolism involving several enzyme systems. 